\title{
Rise, Fall, and Recovery of Blockchains in the Maritime Technology Space
}

\author{
Ziaul Haque Munim ${ }^{1, *}$, Okan Duru ${ }^{2}\left(\mathbb{D}\right.$ and Enna Hirata ${ }^{3}(\mathbb{C}$ \\ 1 Faculty of Technology, Natural and Maritime Sciences, University of South-Eastern Norway, \\ 3184 Horten, Norway \\ 2 Ocean Dynamex Inc., Ottawa, ON K2L 4G7, Canada; okan.duru@oceandynamex.com \\ 3 Center for Mathematical and Data Sciences, Kobe University, Kobe 657-8501, Japan; \\ enna.hirata@platinum.kobe-u.ac.jp \\ * Correspondence: ziaul.h.munim@usn.no; Tel.: +47-99893486
}

Citation: Munim, Z.H.; Duru, O.; Hirata, E. Rise, Fall, and Recovery of Blockchains in the Maritime Technology Space. J. Mar. Sci. Eng. 2021, 9, 266. https://doi.org/ $10.3390 /$ jmse 9030266

Academic Editor: Bruce Hartman

Received: 31 December 2020

Accepted: 23 February 2021

Published: 2 March 2021

Publisher's Note: MDPI stays neutral with regard to jurisdictional claims in published maps and institutional affiliations.

Copyright: (c) 2021 by the authors. Licensee MDPI, Basel, Switzerland. This article is an open access article distributed under the terms and conditions of the Creative Commons Attribution (CC BY) license (https:// creativecommons.org/licenses/by/ $4.0 /)$.

\begin{abstract}
Blockchain technology, since its introduction, has been expected to be implemented in many areas. Cryptocurrency is one unique example that established a functioning application. On the other hand, blockchain technology is not immune to various challenges related to the nature of itself, privacy management, and antitrust laws, among others. This study lays out the nature of blockchain and applications in the maritime industry, while highlighting the bottlenecks. Potential resolutions and anticipated developments are proposed. To do this, we adopt a systematic approach and present an overview of blockchain in maritime literature. In addition, the fundamental problems with blockchain are investigated, beginning from their essentials to the pain points that are claimed to need improvement. For establishing a legitimate and practically meaningful blockchain platform, stakeholders need to achieve pluralism (consensus validation), privacy, and security of the system.
\end{abstract}

Keywords: blockchain; maritime; shipping; internet of things; interoperability

\section{Introduction}

Blockchain is an (open) distributed ledger technology (DLT) that allows data (transactions) to be recorded globally and concurrently on thousands of servers based on a peer-to-peer (P2P) topology, which makes the recording of transactions between two parties efficient, verifiable, and permanent [1,2]. In the fully open (public) blockchain platform, anyone on the network can see everyone else's entries in near real-time, which makes it difficult for one user to manipulate records and gain control of the network [1,2]. In brief, blockchain transforms traditional contracts, transactions, and other valuable records to a digital format and facilitates conventional economic, legal, and political systems to catch up with the speed of the digital world. For gaining the full capacity and productivity of digital space, our traditional authorization and recording structure must be reformed to resonate with the technology of the 21st century.

The debut of blockchain was the application to cryptocurrencies (e.g., bitcoin) [2,3], and the concept has since gained rapid market traction and significant popularity. The first (and still functioning) kind of blockchain executes the public blockchain mechanism, where the fullest transparency is provided to eliminate fraud and gaming of the system, in addition to the processing of authentication operations (validation of digital stamps). A variation of full transparency is achieved by utilizing a cryptology concept called zeroknowledge proof, which is a mathematical method used to verify things without sharing or revealing underlying data [4]. Therefore, zero-knowledge proofs can be used to validate cryptocurrency transactions managed on a blockchain, and eliminate fraud without revealing confidential data about which wallet a payment came from.

In later years, many different kinds of blockchain structures (a.k.a. fabrics) are introduced by developers to expand the use of blockchain in other prospective fields. Private 
blockchains are among those novel variations of the technology [5]. Another layer of features, permission, has been implemented in both public and private blockchains. Ethereum and Bitcoin represent two major public permissionless networks, while Hyperledger Fabric and Quorum are major examples of private permissioned networks. A permissioned network has layers of authority, and only selected participants can join the network $[3,6]$.

Transactions in the maritime industry typically involve many players from different industries from several countries. The very nature of the business makes the industry vulnerable to lack of transparency, and in the process, creates high transaction costs, allows fraud and theft, and is vulnerable to cyber-attack risks, among other problems. Given the promising architecture of blockchains, it could benefit the industry. However, adoption of blockchain has not been very successful in the maritime industry as yet. This study reviews blockchain in maritime literature, identifies the key benefits and challenges, and suggests strategies to overcome the challenges.

While studies exist reviewing the blockchain in maritime literature, this study contributes in three ways. First, this study focuses only on the blockchain in maritime literature; other industries are not included. For example, Jović, Tijan [7] conducted a broader review of blockchain in sustainability, supply chain, and transport, along with maritime industry. Similarly, Dutta, Choi [8] reviewed blockchain in several industries, such as finance, technology, manufacturing, etc., where the focus on the shipping industry is rather brief. Second, unlike previous review studies, we provide a detailed overview of the existing literature using two bibliometric analysis tools-VosViewer [9] and Biblioshiny [10]. Finally, most of the previous review studies only identified benefits of maritime blockchain applications, with some challenges, but this study provides strategies to overcome the challenges.

This study presents blockchain mechanisms in the next section. Section 3 presents an overview of blockchain applications in the maritime industry. Challenges to blockchain implementation are discussed in Section 4, and strategies to overcome the challenges are presented in Section 5. Conclusions are drawn in Section 6.

\section{Principles of Blockchain}

Blockchain technology is initially built upon certain principles that address particular pain points. Those principles are implemented and illustrated in cryptocurrency applications by utilizing a "public" blockchain fabric. A public blockchain network has the complete transparency and visibility of nodes (users), so that no privacy is allowed, in principle [3,5]. Entities can still be hidden behind proxy entities nodes, but transactions are visible to all users. All transactions are executed and stored over multiple nodes (i.e., databases) in the network, so that records of transactions can be retrieved from multiple users $[3,5]$. Those transactions are recorded in an "irreversible" and time-stamped structure where interference and creation of false records are prevented by a consensus validation process [11,12]. All past and present transactions are validated by multiple users through a consensus mechanism. This mechanism implies that the truth (authenticity) is the one on which a consensus is achieved. The consensus feature also requires that a computing capacity of the majority of the network (51\%) can outperform the remainder of the network in completing a block; but the majority may have the power of creating a malicious block, in principle. Since the consensus is achieved by the majority (similar to democratic elections), the majority of validations would have such power. At the same time, this scenario seems quite impractical due to the current size of the network. Finally, all transactions in this fabric are direct, meaning peer-to-peer without a central authority or moderator [11,12]. Transactions are not necessarily required to be monetary; the same fabric can be implemented in official documents or certificates when privacy is not an issue.

Under these circumstances, blockchain networks claim that market intermediaries and regulators are not required to intervene to this structure, as notarization (authenticity validation) can be achieved by complete visibility, and confirmation from multiple nodes $[11,12]$. In practice, this execution process requires millions of computers to work simultaneously to sustain the network and retain a working speed of the network while 
validations are processed. In the cryptocurrency ecosystem, this is achieved by a rewarding system (tokens) that puts incentives for nodes to help the validation mechanism work [13]. The emergence of cryptocurrency mining farms (e.g., Bitcoin mining) is the result of this systemic requirement.

In brief, the nature of blockchain can be identified in the following components:

(i) Peer-to-Peer (direct) transmission: P2P transmission eliminates intermediaries and reduces costs and time.

(ii) Distributed ledger (database records): multiple copies of transactions (in their corresponding order) are stored in the network for security and validation.

(iii) Visibility of network: network must be transparent and visible to all nodes for eliminating fraudulent activities.

(iv) Irreversible and time-stamped records (encryption): certain computational techniques are used to eliminate false records and manipulations. Encryption mechanism is the part of this ecosystem to retain the authenticity of digitally stamped records.

(v) Consensus-based authenticity validation (a.k.a. proof of work): since blockchain is a nonauthority structure, there is no dedicated point of authentication that all users acknowledge. Instead, a consensus-building protocol is executed by using previous features of blockchain (e.g., irreversible records, visibility of network).

While the above mentioned features are relevant for public permissionless blockchain fabrics, variations of blockchain reduce or eliminate some of these features to downsize a public network to permissioned public or private blockchain networks [5]. In other words, some of the principles of blockchain are sacrificed to achieve feasibility for other uses of the blockchain fabric. One of the major modifications of the public blockchain is developed by relaxation of consensus-based authenticity validation. Instead of requiring thousands of nodes to validate a transaction, "Proof of Stake" approach works with pre-identified and authorized validators assigned to blocks [14]. Since a select group of validators executes the process, much higher computing speed is gained. However, this sort of solution raises questions, as an authority is still needed for notarization, which is the pain point that blockchain promises to eliminate at the first place.

Another relaxation can be found in permissioned networks, where only users with permission can join and execute transactions [6]. This kind of structure also needs an authority to define users to approve and customize their visibility and function in the network. With the relaxation of blockchain principles, it is always questioned if the new form of network can be labelled as a blockchain (https:/ / cointelegraph.com/news/why-ibms-blockchainisnt-a-real-blockchain). Permissioned private blockchains (e.g., Hyperledger) discard some major features of blockchain, and therefore, some commentators tend to disqualify them from blockchain space, classifying as a form of digitalization but not really a blockchain. The features of blockchain described above are seen as the "soul" of blockchain, distinctive features that separate from other forms of digital solutions (e.g., digital signature).

In the maritime industry, there exist no applications of the public blockchain so far. There are multiple applications and projects on permissioned blockchains. In the following section, the state of blockchains in the maritime industry and relevant literature are investigated.

\section{Overview of Blockchain Applications in the Maritime Industry}

In the last few years, many developers proposed blockchain networks for various digitalization problems in the maritime industry. Many of these proposals were not more than a pure digitalization of existing systems, and therefore, such attempts did not gain significant market traction. A number of unforeseen issues have been detected, and it was not always easy to overcome those constraints. For example, most blockchains require industry-wide acknowledgement and implementation, which is extremely difficult when there is no binding legislation.

To guide blockchain adoption in the maritime industry, we have reviewed published studies on the topic. The review was conducted in two phases. In the first phase, we 
analyzed published literature on the topic using bibliometric methods and tools. In the second phase, the content of the sample studies was coded for analysis using a literature review matrix in Microsoft Excel. Through the content analysis, we have identified the benefits and challenges of blockchain adoption. Then, considering recent developments, we suggest strategies to make blockchain work in the maritime industry. Figure 1 provides the stages of analysis in this study.

- Most impactful studies.

- Author and country collaboration network. - Four major research themes.

Bibliometric Analysis

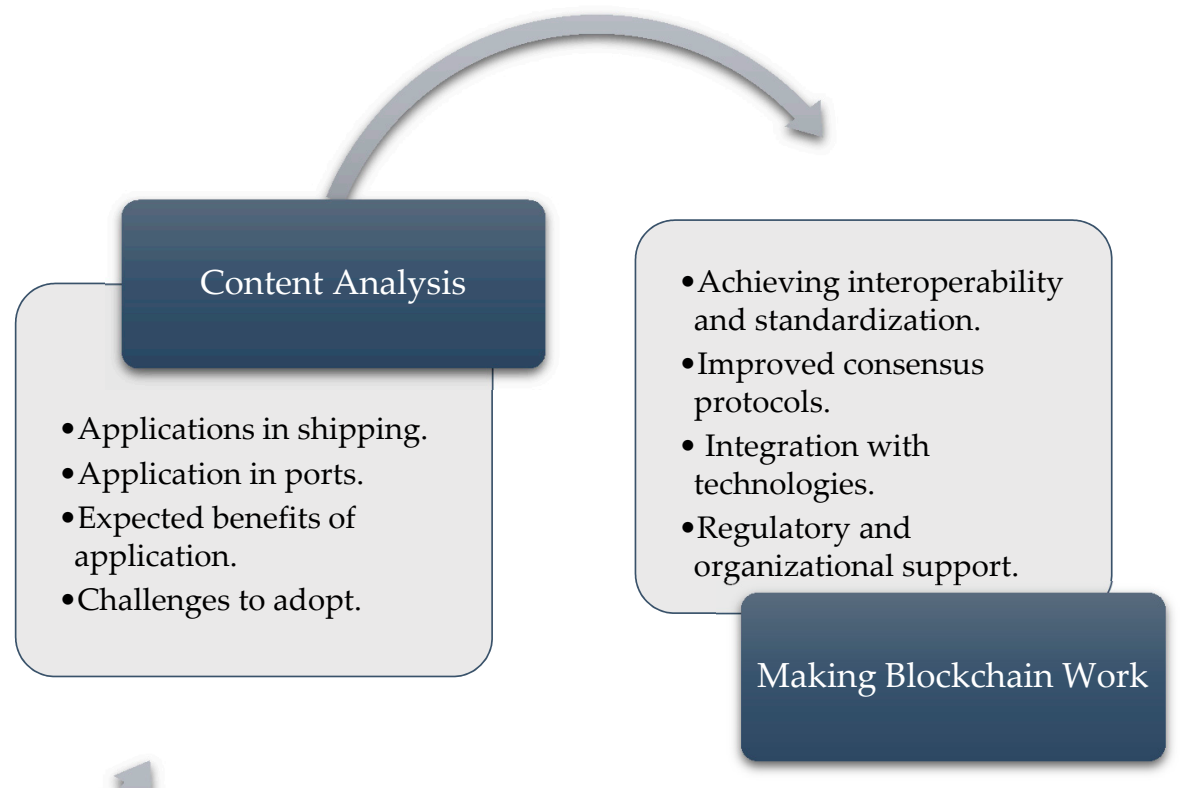

Figure 1. Stages of analysis.

\subsection{Bibliometric Review}

To map developments in the maritime blockchain scene, we adopt a bibliometric review approach. A bibliometric literature review (BLR) can reveal the most impactful studies, sources, underlying research clusters, and more, on a topic such as blockchain in the maritime industry. Bibliography data of published literature were collected through a Boolean search via the Web of Science (WOS) and Scopus database, as reported in Table 1. We proceeded with the Scopus database as it reverts a higher number of records. After limiting to the English language, we had 106 records. We manually screened these studies to confirm their relevance to the topic of blockchain in the maritime. Finally, 48 records were found relevant and extracted for bibliometric analysis, which included 24 articles, 19 conference papers, four reviews, and one book chapter (full list provided as supplementary material). Out of the dropped records, the majority were only introductions to conference proceedings. Some records with the keyword "port" belonged to pure computer science and geographic location names. Other excluded studies only briefly mentioned the TradeLens initiative, a blockchain based initiative of IBM and Maersk, as background information, or a focus on general shipping or the offshore industry, without any maritime involvement. 
Table 1. Blockchain in maritime literature search (WOS refers to the Web of Science database, and the asterisk $\left(^{*}\right)$ in keyword search serves as the wildcard operator).

\begin{tabular}{ccc}
\hline & \multicolumn{2}{c}{ Search Results } \\
\hline Keyword Search (Date of Search: December 13, 2020) & WOS & Scopus \\
\hline ("blockchain*" and "maritime") & 18 & 50 \\
("blockchain*" and ("maritime" or "shipping")) & 31 & 85 \\
("blockchain*" and ("maritime" or "shipping" or "port")) & 35 & 109 \\
Articles after screening manually & - & 48 \\
\hline
\end{tabular}

The 48 sample studies were published in 41 academic outlets (journals, conferences, books) during the last three years, 2018-2020. On average, the sample studies received 4.02 citations, and 2.04 citations per year, per study. In total, 140 scholars authored the 48 studies with an average of 3.04 co-authors per study (only 7 single-authored). Table 2 presents the 10 most cited studies on blockchain in the maritime industry. Total citations (TC) refers to citations received by the articles from all of the articles listed in the Scopus database, as of the date of data extraction. TC per year indicates average citations from the year of publication. These studies are highly recommended for scholars studying blockchain in the maritime industry.

Table 2. Ten impactful studies (ranked by TC, that is, total citations).

\begin{tabular}{ccccc}
\hline No & Study & DOI & TC & TC per Year \\
\hline 1 & Yang [15] & $10.1016 / j . t r e .2019 .09 .020$ & 21 & 10.50 \\
2 & Pedersen, Risius [16] & $10.17705 / 2 m s q e .00010$ & 21 & 10.50 \\
3 & Jabbar and Bjørn [17] & $10.1145 / 3148330.3148345$ & 21 & 7.00 \\
4 & Tan, Zhao [18] & $10.4018 /$ IJISSCM.2018040103 & 19 & 6.33 \\
5 & Hasan, AlHadhrami [19] & $10.1016 /$ j.cie.2019.07.022 & 15 & 7.50 \\
6 & Czachorowski, Solesvik [20] & $10.1007 / 978-3-030-00253-4 \_24$ & 15 & 7.50 \\
7 & Allen, Berg [21] & $10.1002 /$ app5.281 & 11 & 5.50 \\
8 & Xu, Chen [22] & $10.1109 /$ THS.2018.8574184 & 9 & 3.00 \\
9 & Bavassano, Ferrari [23] & $10.1016 /$ j.rtbm.2020.100428 & 7 & 7.00 \\
10 & Wang and Qu [24] & $10.1007 / 978-981-13-8683-1 \_23$ & 7 & 3.50 \\
\hline
\end{tabular}

Bibliometric tools can be used to visualize collaboration networks within a topic. Figure 2a,b present an author and country collaboration network, respectively, within blockchain in maritime research. We notice two (yellow and green clusters) strongly connected groups of scholars in Figure 2a. Jović M. and Tijan E. appear larger with a stronger node as they co-authored two of the sample studies, i.e., Jović, Tijan [7] and Jović, Filipović [25]. On the country level collaboration, scholars from European countries seem to collaborate strongly, having the United Kingdom as the center (violet cluster). A similar pattern is observed among scholars from Asian countries (green cluster). Although Croatian scholars published multiple studies in the topic, they have not collaborated with scholars from other countries so far. 


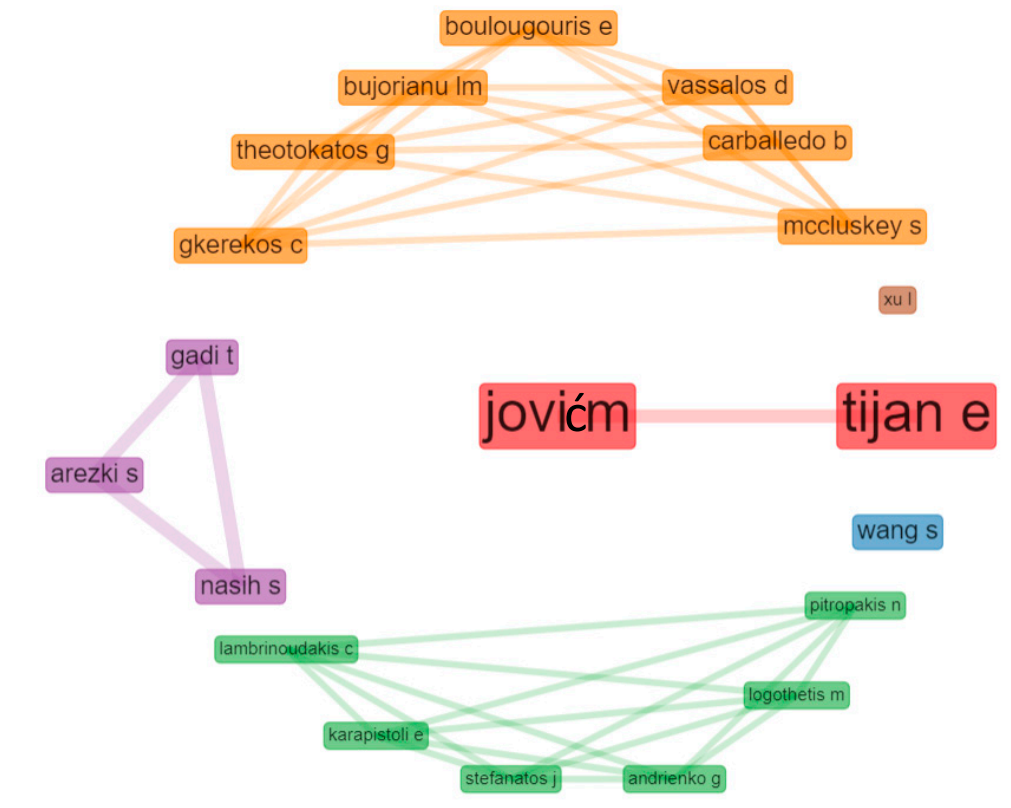

(a) Author collaboration network

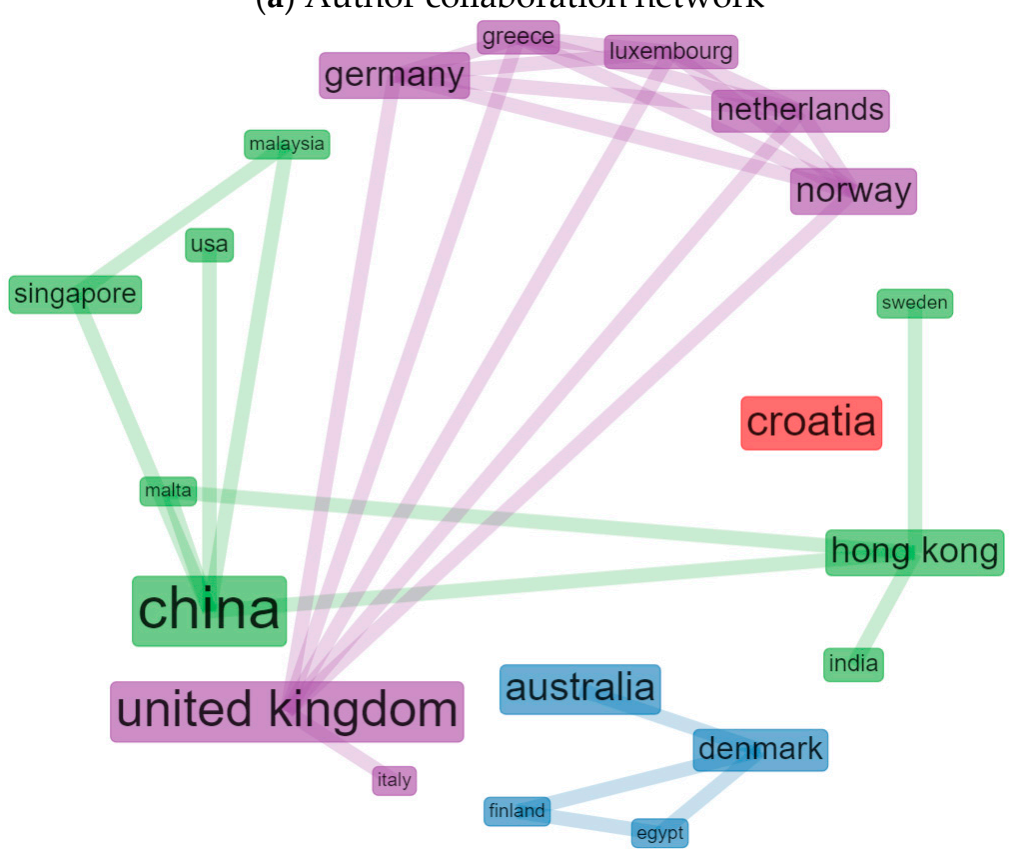

(b) Country collaboration network

Figure 2. Collaboration network visualization (Louvain clustering algorithm of 20 nodes using star network layout in Biblioshiny software).

To map the blockchain in maritime literature, we used keyword co-occurrence analysis. The analysis included only author keywords and keywords plus assigned by the Scopus editors. We used the VosViewer software [9] for analysis with the threshold of minimum of two keyword co-occurrence. The keyword co-occurrence network in Figure 3 indicates four major themes: (1) blockchain applications in maritime (blue cluster), (2) challenges in blockchain applications (red cluster), (3) blockchain technologies (yellow cluster), and (4) blockchain adoption (green cluster). These four research themes were used to categorize the 48 studies in the literature review matrix. 


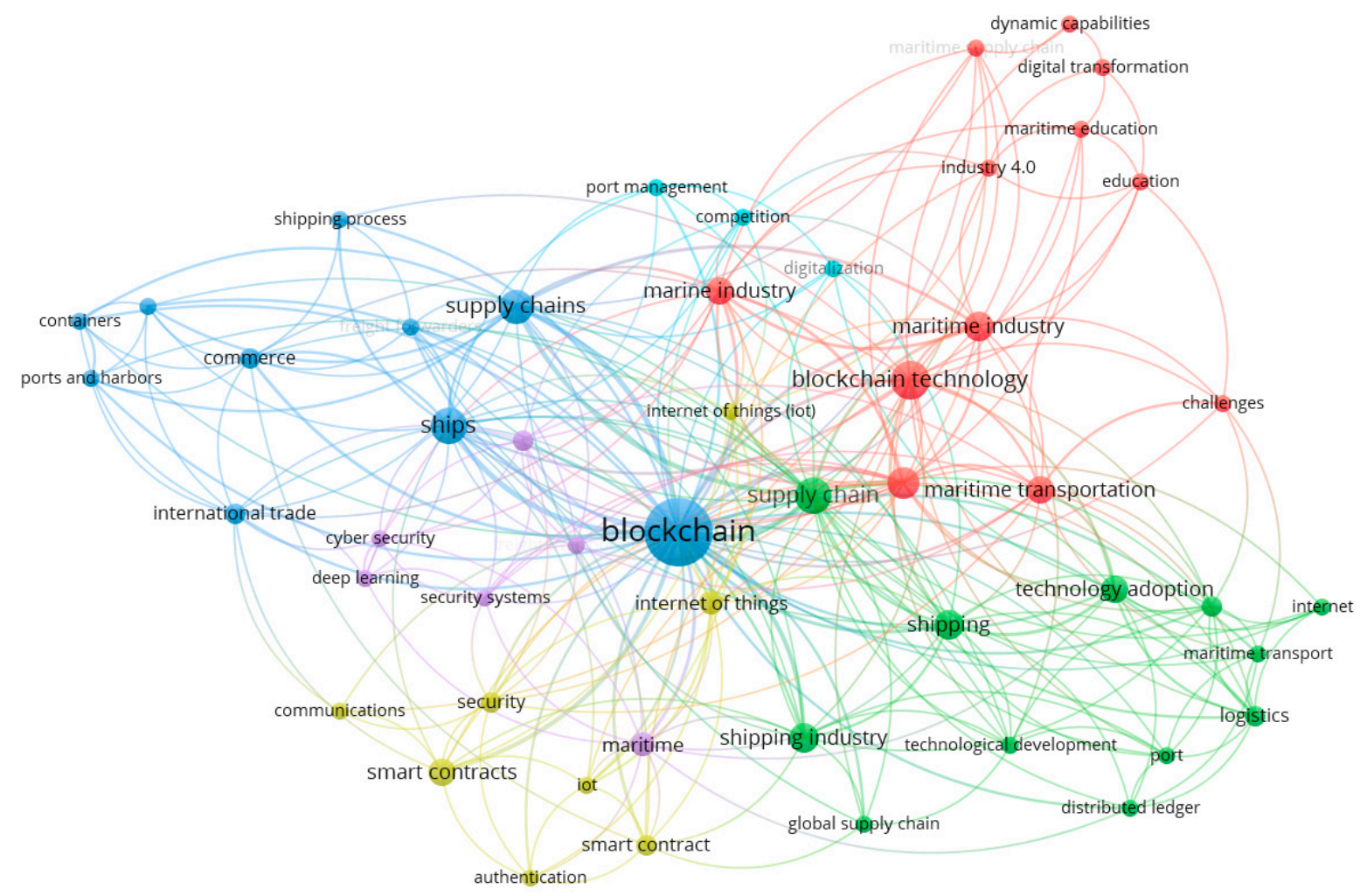

Figure 3. Keyword co-occurrence network (using VosViewer software with a minimum of two co-occurrences, resulting in 51 keywords).

\subsection{Blockchain Applications in the Maritime Industry}

\subsubsection{Applications in the Shipping Industry}

The sample studies focused on maritime applications of blockchain technology. The most promising application of blockchain is expected to digitize the container shipping and third-party logistics business - the electronic bill of lading in particular [15,26-28]. A number of consortiums have initially committed to delivering a blockchain solution in the container shipping business, including Cargo Smart and IBM's TradeLens [25,29]. TradeLens specifically offers a Hyperledger fabric mainly funded and led by Maersk Line and IBM. Furthermore, blockchain applications in shipping include smart contracts for charter party contracts [30], freight booking and consolidation operation [18,31], real-time cargo tracking [22], etc.

Blockchain technology can be a solution for tracking products and ensuring the originality feature. For example, Hasan, AlHadhrami [19] proposed an Ethereum blockchainbased smart containers with the internet of things (IoT) sensors for tracking location, and monitoring temperature, air pressure, humidity, and other relevant attributes. Similarly, marine bunker fuels may be monitored with a blockchain-based tracking system. Fuel quality, contaminations, grade mismatch (e.g., sulfur content), and some other features of marine bunkers are difficult to follow-up by users (i.e., ship operators) other than trusting major suppliers. Particularly the sulfur cap regulation (IMO2020) has led to concerns as the violation of rules may be penalized by port states.

Another promising development relates to certification authorities, such as flag states and classification societies [20]. Both ship and seafarer certificates are expected to be converted into a blockchain-based authentication process where the authenticity of claimed approval is validated through the common blockchain network. Since those certificates are compulsory for ships and seafarers, all operating ships and their crews, must be intrinsically certified, which is not private information at all. The nature of certifications allows much easier implementation compared to other uses consisting of commercial interests. 
Further, Peronja, Lenac [27] show an expected reduction in container freight rates as a result of blockchain application in shipping companies. Similarly, Tan, Zhao [18] proposed an export platform that can optimize less than container load (LCL) shipments for international shipping by sharing shipment information among freight forwarders and shippers. Moreover, to demonstrate blockchain applications in shipping, Komathy [32] presented a proof-of-concept of the distributed blockchain shipping (DBCS) architecture that brings real-time international shipping activities, including banking, manufacturing, and insurance under a secured IoT system. Finally, applications of blockchain technology in maritime autonomous surface ship (MASS) operations have been investigated [33-35].

\subsubsection{Applications in Ports}

Ports around the world have been initiating blockchain implementations as well. Extant literature shows blockchain initiatives at the Port of Long Beach [36], Port of Rotterdam [37], and some Indonesian ports [38]. The proposed application at the Port of Long Beach shows improved energy management at the port while reducing cost and improving security [36]. The main application of blockchain technology at ports seems to be to improve the efficiency of port traffic management [37-39], including container stacking [40]. Blockchain-based technologies at ports are likely to reduce bureaucracy in port operations (particularly in developing countries) by improving the visibility of overall port traffic, and the use of built-in data analytics can help reduce port turnaround time.

Keeping aside the literature review type studies (for example, $[7,8,15]$ ), in the sample of 48 studies, Table 3 summarizes the key benefits of blockchain applications in the maritime industry.

Table 3. Benefits of blockchain in maritime.

\begin{tabular}{cc}
\hline Expected Benefit & Source \\
\hline Transparent maritime supply chains; immutable and infallible record & {$[31,41,42]$} \\
Intermediary cost reduction & {$[42-44]$} \\
Time efficiency & {$[41,42]$} \\
Reduced bribery, fraud, and theft & {$[30,41,43]$} \\
Digital document sharing and signing; smart contract; electronic bill of & {$[21,26-28,30,31,41]$} \\
lading; paperless trade; efficient customs clearance & {$[36]$} \\
Efficient energy management & {$[36,41,42]$} \\
High-level security system & {$[36]$} \\
Optimized system maintenance cost & {$[33,35]$} \\
Improved port traffic management & {$[39]$} \\
Improved container utilization & {$[30]$} \\
Reducing transaction costs & {$[19,22,43]$} \\
Supply chain emissions reduction & {$[43,45]$} \\
Reduced container freight rate & {$[27]$} \\
Optimize container load, container stacking & {$[27]$} \\
Accelerate bureaucratic process & {$[18,40]$}
\end{tabular}

\section{Challenges in the Blockchain Applications}

In contrast to various advantages of blockchains, the technology requires a significant reformulation in the digital systems, and users need to tolerate some aspects that would be avoided traditionally. Accordingly, most applications demand a mediation process to satisfy users' requirements while retaining the fundamental features of blockchains.

\subsection{Lack of Authority for Standardization}

One of the major challenges in blockchain implementation in the maritime industry is the lack of standardization in data elements $[7,28]$. In addition, a key restraining point lies in the fact that none of the international governing bodies assume authority and 
responsibility about the blockchain development [7]. Blockchain solutions are unregulated and voluntary systems in nature. For example, the massive Bitcoin market is neither regulated nor inspected by an authority. Instead, most regulators object to cryptocurrencies. Expecting an authority over an unregulated (or self-regulatory) system seems counterintuitive as the pain point is the loss of time and transaction costs with the traditional regulated systems.

A regulatory vacuum stands as the essential challenge with blockchain development. The Digital Container Shipping Association (DCSA) has emerged in this circumstance to establish a self-regulatory framework in the industry. DCSA commits to developing standards and a blueprint of progress to achieve the ultimate goal of practical and viable use of the technology.

\subsection{Interoperability and Lack of Scale}

Different stakeholders in the maritime industry, such as banks, freight forwarders, ports, etc., use different systems, which make data exchange among the stakeholders cumbersome and almost impossible to achieve interoperability among their systems [41]. It was expected that blockchain technology would facilitate interoperability. In this notion, during the first emergence of blockchain, most firms have attempted to own a dedicated blockchain. Some consortiums even assumed that the proposed solution would be a de facto standard so that most competitors would acknowledge its superiority and join the consortiums. However, this assumption certainly did not work. Because of the development of multiple solutions, the interoperability problem (permeability of blockchain data across platforms) has arisen in several uses [21,46]. Liner shipping is one specific example of the problem, which is addressed by DCSA. Considering the nature of liner shipping alliances, interoperability is a hard requirement for meaningful use of blockchain technology. In relation to interoperability, there are not many users in most maritime applications [47]. Thus, the consensus principle is not relevant in a permissioned private networks [26]. That is also a debate if we could classify such digitalization as a blockchain. Under the limited number of users, a major deviation from the essential feature of blockchain becomes an unavoidable fact.

\subsection{Antitrust Law and Commercial Privacy}

Most legislations on competition require a boundary of communication between competitors to eliminate any antitrust structures. The visibility and transparency features of public blockchains and permissioned private blockchains (for permissioned users) violate the legal requirements [25], and therefore, applications in commercial documents need modifications and restructuring [26]. In the context of liner shipping, data on shared spaces may be exchanged between firms while broader visibility is not allowed. End-toend encryption schemes (public-private keys) must be embedded to the system with the capacity of defining certain nodes (e.g., a specific bill of lading) to be shared bilaterally. There are developments on this issue and these will be discussed in Section 5 .

\subsection{Environmental Concern}

Although it is not a significant issue for permissioned blockchains (dedicated validators in place), public permissionless blockchains require massive computational work, as in mining farms. Since the maritime industry does not intend to use such gigantic networks, environmental concern is not a priority bottleneck for the industry, but it is a general issue with certain types of blockchains [7].

\subsection{Dispute Resolution}

The unregulated nature of blockchains raises questions about potential disputes on commercial affairs [26]. This problem will most probably be resolved with underlying legal papers (a digital document convertible for legal purposes) while the disputes regarding the technology, data, and other electronic means may need subject matter jurisdiction 
(specialty courts). The preparedness of courts and legal systems is a bottleneck for the technology [30].

\subsection{Data Tampering and Hacking}

While studies argue that blockchain provides hacking-free high-level security $[8,36,41]$, a number of hacking events significantly deteriorated the reputation of the blockchains (https: / / www.technologyreview.com/2020/09/10/1008282/north-korea-hackers-moneylaundering-cryptocurrency-bitcoin/). In permissioned private blockchains (the majority in the maritime), the validation process is assigned to certain users similar to the traditional structure, and therefore, the risk of hacking is negligible with this framework. Most applications in the maritime do not execute cryptocurrency or other cash equivalents. Zero risk may not be claimed while the existence of underlying physical assets (e.g., containers) reduces the risk comparing to the non-asset cryptocurrencies $[48,49]$.

\section{Major Resolutions to Challenges}

The challenges of blockchain applications in the maritime industry have been discussed in the previous section. Making blockchain work is, to a large extent, related to how to overcome those challenges. Due to long transaction times, as well as huge energy consumption, permissionless (public) blockchain is not considered suitable for application in business fields including shipping. Unless otherwise specified, the blockchain discussed in this section is for permissioned (private or consortium) blockchain, e.g., HyperLedger, Corda, and Ethereum. The following sub-sections discuss blockchain adoption in maritime in detail, from technology and non-technology perspectives.

\subsection{Technology Perspective}

\subsubsection{Interoperability}

Interoperability is one of the top technical challenges for blockchain application across industries [8]. Interoperability means communication and data exchange between two or more different applications. Additionally, interoperability of blockchain involves reading, monitoring, and acting on states and events [50]. Research on blockchain interoperability distinguishes three types of technologies [51].

(1) Notary schemes: where a party or a group of parties agree to perform an action on ledger B given some event occur on ledger A.

(2) Relay (sidechain) schemes: where systems within one blockchain can read and validate events and/or state in other blockchains.

(3) Hash-locking: where two ledgers can read from each other. For example, ledger A can read from ledger $\mathrm{B}$, and vice-versa. This allows a swap of tokens from ledger A to ledger B.

Interoperability can take three forms, namely, interoperability with (non-blockchain based) legacy systems, interoperability with another blockchain ledger (interchain), and interoperability between different applications in a single ledger (intrachain). In this study, we focus on the interchain and intrachain operability, since the interoperability with legacy system is not of major concern thanks to application programming interface (API) technology.

Enabling interoperability is important to shipping since the transactions are handled in different blockchains in most of the cases. For example, supply chain transactions may take place in more than one regional blockchain. Moreover, tokenized digital money may be used for payment on a separate blockchain. Brown [52] proposes five technical stages of blockchain interoperability (Figure 4). In the first stage, blockchain is able to integrate with existing legacy systems. Second, blockchain is able to initiate transactions with other networks. Third, one blockchain is able to interact with other blockchains of the same technology, e.g., a Hyperledger-based blockchain to do a transaction with another Hyperledger-based blockchain. Fourth, a blockchain (e.g., Hyperledger-based) is able to 

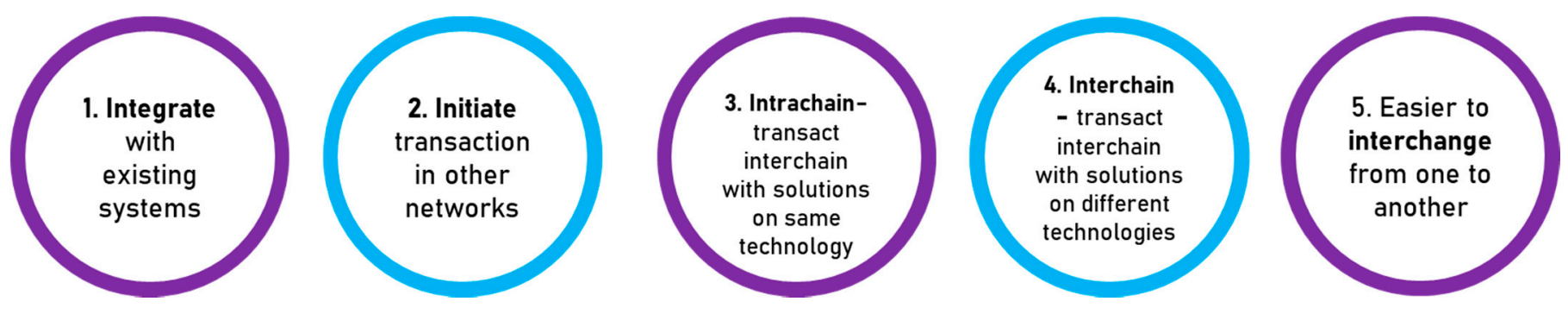

Figure 4. Five stages of blockchain interoperability. Source: authors compiled based on Brown [52].

An example of intrachain interoperability is Corda Distributed Applications (CorDapp) provided by R3, an enterprise software firm, using its Corda network. Corda's solution to interoperability introduces a shared underlying network on which private networks can be built, which is based on a notary scheme. Participants not only create and maintain private networks, but also interact with other private networks that exist on the shared network through the CorDapp. The goal of a CorDapp is to allow nodes to reach agreement on updates to the ledger [53]. With the solution, participants are able to exchange data with other participants on the Corda Network, without having to share their private networks. The scenario is, however, an intrachain solution-it works only when the participants who want to transfer data are all on the Corda Network.

An example of interchain interoperability is the Cactus project led by Accenture and Fujitsu, which aims to enable faster asset transfers as well as streamline the recovery process of transaction errors in the blockchain. The HyperLedger Cactus uses Fujitsu's proprietary security technology "ConnectionChain" and Accenture's "Blockchain Integration Framework". Accenture and Fujitsu [54] proposed a solution to establish a trusted "interoperability node" (Figure 5). The concept is to merge two or more blockchain networks, rather than transferring the data from one to another, which is implemented as relay scheme.

DLT 1

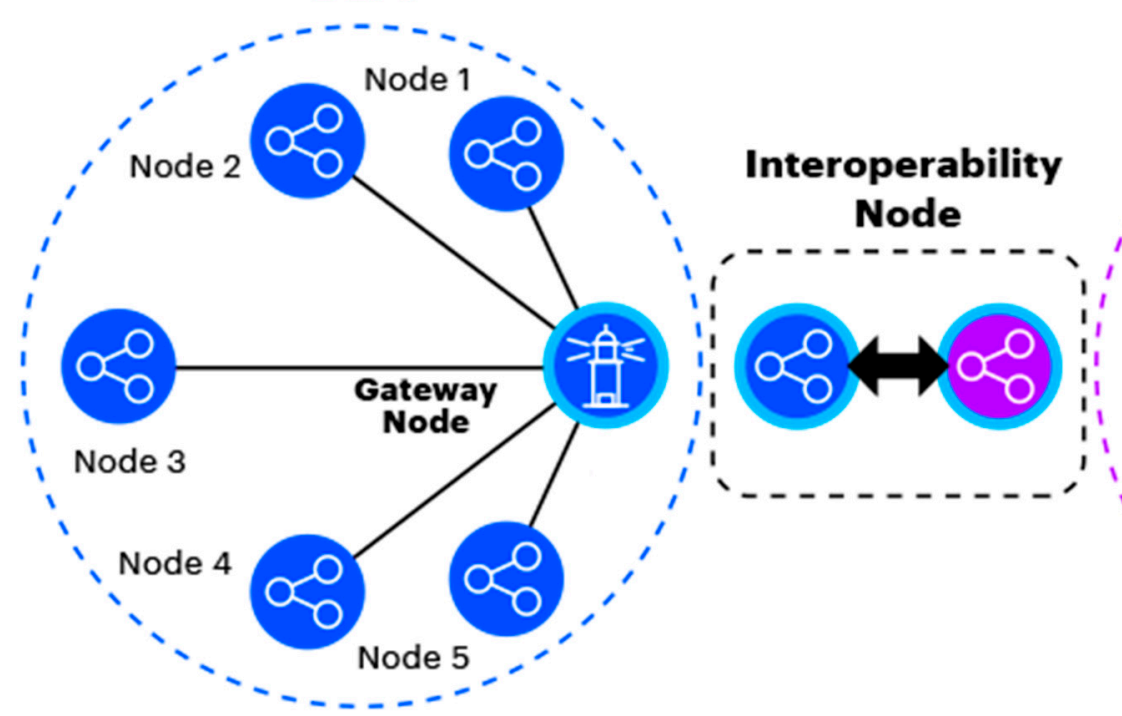

DLT 2

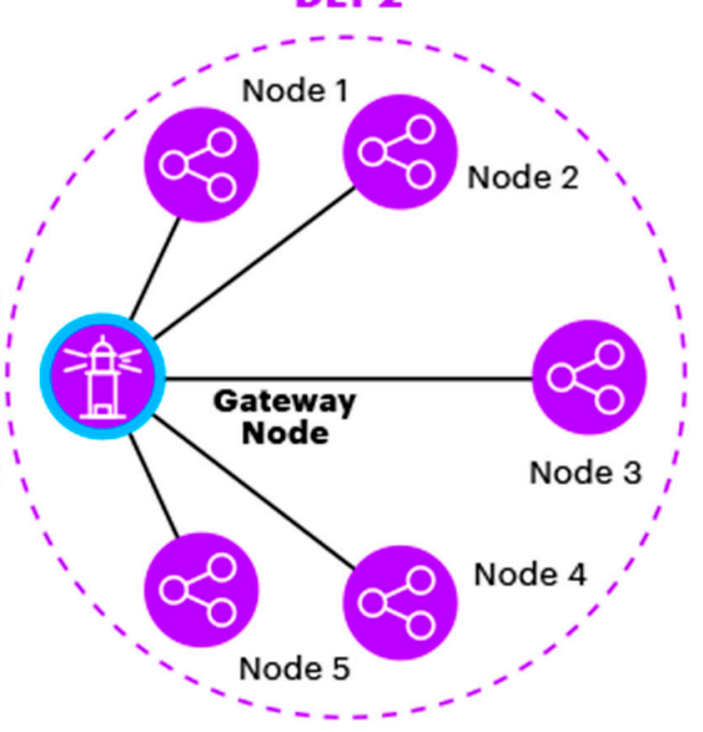

Figure 5. A trusted "interoperability node". Source: Accenture and Fujitsu [54].

HyperLedger Cactus supports Hyperledger Fabric, Quorum, R3's Corda, and enterprise Ethereum client Hyperledger Besu. Since Hyperledger Besu is capable of being used in the public blockchain, Cactus is considered to be able to handle the integration of both 
private and public blockchains. In addition to the examples discussed, other solutions, such as 2-step blockchain construction and on-chain off-chain, are also being proposed to enable interoperability and avoid data explosion. However, none of them represents a perfect solution. Similar to Kaska and Tolga [55], multi-criteria decision-making (MCDM) frameworks can be used for the selection of appropriate blockchain technology.

Interoperability is more a matter of designing a solution that balances security, governance, adaptability, and economic incentives that suit their target market [56]. These goals are being achieved in the latest blockchain protocol Avalanche. We will discuss Avalanche in sub-Section 5.1.3.

\subsubsection{Standardization}

Standardization is not only one of the fundamental requirements for shipping to go digital [15], but also a key criterion of enabling interoperability [57]. Each of the different technologies, HyperLedger, Corda, Ethereum, is fundamentally different in how they define permissions, so translating from one to another is risky [58].

Although open-sourced blockchain technologies do not care about standardization, many organizations, including international and regional standard bodies, are working on the standardization of blockchain [59]. International bodies include the International Organization for Standardization (ISO), the International Telecommunication Union (ITU), the World Wide Web Consortium (W3C), the Institute of Electrical and Electronics Engineering (IEEE), the Internet Engineering Task Force (IETF), the Internet Research Task Force (IRTF), Organization for the Advancement of Structured Information Standards (OASIS). Regional organizations include Standards Australia (SA), the International Securities Association for Institutional Trade Communication (ISITC) Europe, the Research ANd Development (RAND), the European Committee for Standardization (CEN), and the European Committee for Electrotechnical Standardization (CEN-ELEC), The European Telecommunications Standards Institute (ETSI), the National Institute of Standards and Technology (NIST), the United Nations Economic Commission for Europe (UN-ECE), etc. All of these organizations are devoting efforts in standardizing blockchain. Notably, these standards are heavily biased towards Europe, and North America. Latin America, Africa, and Asia should also play a role in the standards decision process.

While multiple organizations are devoting to blockchain standardization (Table 4), blockchain has been implemented in various industries, and most of these industries have their own standard existing already. This is also the case of shipping and logistics. Members of Blockchain in Transport Alliance (BiTA) are working to develop blockchain standards for the industry, while ISO also aims to release a series of blockchain and distributed ledger technology (DLT) standards no later than 2021. This could potentially result in duplicates or even conflicts of standard.

Table 4. Classification of international and regional standardization.

\begin{tabular}{cccccc}
\hline & International & Regional & Governmental & Industrial & Open Source \\
\hline ISO TC307 & $\mathrm{X}$ & & $\mathrm{X}$ & & \\
ITU-T & $\mathrm{X}$ & & $\mathrm{X}$ & & \\
CEN & & $\mathrm{X}$ & $\mathrm{X}$ & & \\
CENELEC & & $\mathrm{X}$ & $\mathrm{X}$ & \\
ETSI & $\mathrm{X}$ & & $\mathrm{X}$ & \\
IEEE & $\mathrm{X}$ & & $\mathrm{X}$ & \\
W3C & $\mathrm{X}$ & & $\mathrm{X}$ & $\mathrm{X}$ \\
IETF/IRTF & $\mathrm{X}$ & & $\mathrm{X}$ & $\mathrm{X}$ \\
OASIS & $\mathrm{X}$ & & & \\
BiTA & & & & \\
\hline Source: Authors compiled partially based on European Commission [60].
\end{tabular}

Standardization is not only critical for interoperability, but also important for the reduction of security risks and vulnerabilities. Standard technical implementations and 
architectures are needed. In addition, standardization is also critical to governance. Governance is related to agreeing who has what powers, rights, and responsibilities. In the context of blockchain, it is also a question of decentralizing power while leaving room for community collaboration.

With the rapid adoption of blockchain technology, standard technical implementations and architectures are needed. When we have too many standards in place, a challenge arises, i.e., how to decide which one will overrule. The good news is that organizations such as the International Association for Trusted Blockchain Applications (INATBA), established in 2019, has launched the process to converge dialogue around standards with relevant bodies including governmental advisories, and private sectors.

\subsubsection{Consensus Protocols (Balance of Scalability, Latency, and Governance)}

Consensus protocol is a technology that defines the performance and security of a blockchain [12]. The main task of a consensus protocol is to confirm a common transaction by all participating nodes in the blockchain. Typical consensus algorithms include Proof of Work (PoW), Proof of Stake (PoS), Delegated Proof of Stake (DPoS), Byzantine Fault Tolerance (BFT), etc. $[4,12]$. The basis on features of consensus, blockchain is categorized as Classical protocol, Nakamoto protocol, and Avalanche protocol [61], see Table 5.

Table 5. Comparison of consensus protocols.

\begin{tabular}{cccc}
\hline Features & Nakamoto & Classical & Avalanche \\
\hline Robust & Yes & No & Yes \\
Low latency and & No & Yes & Yes \\
quick finality & No & Yes & Yes \\
High throughput & No & Yes & Yes \\
Lightweight & No & Yes & Yes \\
Low energy & No & No & Yes \\
Cansumption & No & No & Yes \\
adversaries & & &
\end{tabular}

Source: Sirer [62].

Comparing to existing protocols, the BFT-based Avalanche protocol works without precise membership knowledge, hence is highly efficient and robust, and can achieve high throughput and quick finality. In the classical protocol, every node needs to talk to every other node. However, in the avalanche protocol, every node talks to a small subset (validator) for a small number of rounds. This dramatically reduces the latency to 1/3600 comparing to Bitcoin; consequently, it can achieve the performance of 7100 transactions per second, which is three times as fast as the transaction speed of Visa, compared to 5 for bitcoin [62].

Most of the existing blockchain platforms cannot support the trade of digital assets at a global scale, and all of the networks offer the same, least common denominator service. Sustainable blockchain needs to fix these issues, while having the ability to evolve with the changing needs of users for the sake of governance. Future blockchain platforms need to be able to support multiple virtual machines, multiple scripting languages, and full control over the lifecycle of digital assets.

The Avalanche protocol enables scalability, usability, flexibility, and governance, which makes it one of the most promising platforms of blockchain. Avalanche is initially built for serving financial markets. While all (both permissionless and permissioned) blockchains in the Avalanche ecosystem can be interoperable with each other, it may be quickly adopted and implemented in other industries than finance. This would form a successful use case of a combination of public and private blockchains. 


\subsubsection{Integration with IoT and Other Technologies}

Integration of blockchain and IoT helps strengthen security, privacy, and governance amongst participants [63]. As mentioned earlier, Komathy [32] presented a Proof of Concept (PoC) to control and configure IoT devices by using blockchain that provides maritime players real-time visibility of all transactions. Blockchain can be used to set up a decentralized digital platform for IoT data that can be accessed by multiple parties as per data governance. Hasan, AlHadhrami [19] proposed an IoT-sensor enabled blockchain system for tracking and monitoring of smart containers that support automated payments through smart contracts. Such IoT based systems are reliable and secure [41].

Artificial intelligence (AI) could be applied in a blockchain platform to deliver bug-free smart contracts, for example, in case of violations of conditions encoded in a smart contract, the system would refund payment automatically [19]. Further, AI-enabled blockchain applications are evident in autonomous ship operations [33-35]. The convergence of blockchain, IoT, and AI technologies, as such, will drive innovative business model developments and digital transformation across these industries.

Meanwhile, according to Nguyen, Chen [48], the erroneous data reporting happened outside of blockchain is one of the most connected risks. Reporting of erroneous data hinders the main purpose of blockchain, which is, protecting data accuracy. Depending on the level of integration, instances of erroneous data may lead to accumulative damages. Integration with IoT and AI could help reduce the management of erroneous entries outside of the blockchain, which has been a major pain point, since it is not something the blockchain technology itself can control. Yet some risk of erroneous data input remains as IoT sensors might report false readings occasionally.

\subsection{Non-Technology Perspective}

\subsubsection{Regulation}

There is debate on whether blockchain actors should mirror traditional corporate law and corporate governance mechanisms for ensuring legal clarity [60]. Despite the ideal of decentralization in blockchains, it is not achievable yet in reality, especially in the case for private blockchains, where traditional governance structures are implemented [26].

The smart contract is a method to bring immutability and transparency in the contractual processes. It is not a legal contract, but nothing more than a piece of code. A glitch in the codes could lead to the misconduct of a smart contract. While a smart contract should be codified with considerable accuracy and precision to preserve the legal bindings of existing contract laws, it calls for a new set of regulations that rule smart contract in particular.

Several existing global regulations impact blockchain. One example is the European Union's (EU's) General Data Protection Regulation (GDPR), which provides that right to be forgotten, i.e., as per article 17, the users have the right to "obtain from the controller the erasure of personal data" [64]. This causes a big challenge for blockchain due to its immutability. In addition, there exist two EU directives based on G20 requirements-payment services directive and an anti-money laundering directive. From the legal perspective, the impact of the two directives on smart contracts is an issue to be addressed. Implementation of blockchain in shipping is also subject to the resolution of issues discussed [23]. This may not likely happen before standardization is achieved.

\subsubsection{Organization Readiness and Adoption Model}

Despite the fact that the number of developers contributing to enterprise blockchain have multiplied by 12 in the past three years [65], the level of blockchain technology-related competence and knowledge of stakeholders in the maritime industry is of general concern [66,67]. Adopting a Technology Acceptance Model (TAM), Yang [15] investigated the intention to use blockchain technology in the maritime supply chain. The result indicates that older people (in many cases in senior positions) are less interested in new technology. The author suggests managers allocate resources to in-depth study of blockchain appli- 
cation and adoption areas to gain competitive advantages. Scholars work to explore the application of management theories and blockchain adoption (Table 6).

Table 6. Management theories and their effects in blockchain adoption.

\begin{tabular}{|c|c|c|}
\hline Theory & Definition & Effects on Blockchain Adoption \\
\hline Principal Agent Theory (PAT) & $\begin{array}{l}\text { Theory of interaction between a principal } \\
\text { and an agent who act on principal's } \\
\text { behalf, focusing on incentives structure in } \\
\text { a way that encourage the agent to act for } \\
\text { the benefit of the principal [68]. }\end{array}$ & $\begin{array}{l}\text { Agents selection and smart contract } \\
\text { design [69]. }\end{array}$ \\
\hline Transaction Cost Economics (TCE) & $\begin{array}{c}\text { Originally applied to "make vs. buy" } \\
\text { vertical decision. Now expanded to a } \\
\text { broad range of organizational } \\
\text { phenomena [70]. }\end{array}$ & $\begin{array}{l}\text { Minimize transaction cost by reducing } \\
\text { cost for information searching, } \\
\text { negotiation, and decision-making [71]. }\end{array}$ \\
\hline Resource Based View (RBV) & $\begin{array}{l}\text { A managerial framework proposed by } \\
\text { Barney [72], is often used to determine } \\
\text { the strategic resources a firm can exploit } \\
\text { to achieve sustainable } \\
\text { competitive advantage. }\end{array}$ & $\begin{array}{l}\text { All relocation of resources to create new } \\
\text { areas of competitive advantages [8]. }\end{array}$ \\
\hline
\end{tabular}

Using an MCDM framework, Zhou, Soh [66] studied the critical factors influencing successful blockchain adoption in the maritime industry. Apart from regulatory support, capital investment, staff training, support from senior management and shipping community are of the highest importance. Hence, the opportunity lies in areas such as the provision of blockchain training services, and provision of ready-made containerized integration packages (for example, a Docker container). The availability of an implementation roadmap is also critical to achieving successful adoption of blockchain technology.

There is no doubt that adoption of blockchain is also subject to affordable costs, including both implementation costs and operational costs. Currently, the costs of blockchain technology are highly variable and unpredictable $[23,43]$. A containerized solution can help standardize and transparentize the implementation cost.

\section{Conclusions}

Blockchain technology provides a new form of security and allows trustworthy anonymous communication to take place in a multitude of areas around the world. The integration of blockchain with other innovative technologies, such as IoT and AI, will improve the efficiency in multiple industries, and even the quality of human life. This study presents an overview of the blockchain applications in the maritime industry using bibliometric analysis tools and methods. We discussed the blockchain applications, benefits, challenges, and strategies to overcome the challenges. A wide range of applications can be observed in the maritime industry, from energy management at ports to autonomous ship operations (see Section 3.2). The major benefits include transparency of the maritime supply chain, cost and time efficiency, digital document sharing and signing, and reducing bribery, fraud, and theft (see Table 3). Achieving interoperability and standardization are two main implementation challenges. To overcome the challenges, a sound regulatory framework, training of employees, and top management support are crucial.

Standardization is critical for enabling interoperability, reducing security risks and vulnerabilities. It is also critical to governance, which is, related to agreeing who has what powers, rights, and responsibilities. In the context of blockchain, it is also a question of decentralizing power while leaving room for community collaboration. Given multiple organizations at the industry, country, regional, and international level are devoting much effort to blockchain standardization, it calls for actions to converge dialogues around standards.

Integration with IoT and AI will drive business model innovation as well as a digital transformation across the maritime and other industries. From a regulation perspective, 
a new set of regulations is urgently needed that provides the right to be forgotten, and also needs to be resolved. Besides, the availability needed to govern smart contracts. The conflict with existing global regulation, such as EU's GDPR of a blockchain implementation map, training services, affordable implementation, and operation costs are also of high priority for the maritime industry to embrace blockchain.

We are living in a world full of networks and databases for different business requirements. The most critical thing is to understand how to catch up with the rapid pace of change. Given there are a great variety of business use cases and requirements, it is not possible to have one blockchain solution to rule all. However, a platform like Avalanche could potentially act as a marketplace of blockchains that could optimize security, interoperability, scalability, latency, and governance, and consequently provide a solution for smooth adoption of blockchain. Hence, more evidence-based (both qualitative and quantitative) studies are expected. Future studies should emphasize documenting blockchain application failures in the industry, learnings from failures, exploring adoption dynamics, measuring the impact on business model innovation, and training maritime professionals.

Supplementary Materials: The following are available online at https:/ / www.mdpi.com/2077-131 2/9/3/266/s1, Excel File S1: List of 48 sample studies.

Author Contributions: Conceptualization, O.D.; methodology, Z.H.M.; software, Z.H.M.; validation, O.D. and E.H.; writing-original draft preparation, O.D., E.H., and Z.H.M.; writing-review and editing, O.D., E.H., and Z.H.M. All authors have read and agreed to the published version of the manuscript.

Funding: Ziaul Haque Munim acknowledges support from the MARKOM 2020 project of the Norwegian Government.

Institutional Review Board Statement: Not applicable.

Informed Consent Statement: Not applicable.

Data Availability Statement: Data provided as supplementary material.

Conflicts of Interest: The authors declare no conflict of interest.

\section{References}

1. Morkunas, V.J.; Paschen, J.; Boon, E. How blockchain technologies impact your business model. Bus. Horiz. 2019, 62, 295-306. [CrossRef]

2. Hughes, A.; Park, A.; Kietzmann, J.; Archer-Brown, C. Beyond Bitcoin: What blockchain and distributed ledger technologies mean for firms. Bus. Horiz. 2019, 62, 273-281. [CrossRef]

3. Lewis, R.; McPartland, J.; Ranjan, R. Blockchain and financial market innovation. Econ. Perspect. 2017, $41,1-17$.

4. Zhang, R.; Xue, R.; Liu, L. Security and privacy on blockchain. Acm Comput. Surv. (Csur) 2019, 52, 1-34. [CrossRef]

5. Buterin, V. On public and private blockchains. Ethereum Blog 2015. Available online: https://blog.ethereum.org/2015/08/07/onpublic-and-private-blockchains / (accessed on 20 November 2020).

6. Polge, J.; Robert, J.; Le Traon, Y. Permissioned blockchain frameworks in the industry: A comparison. Ict Express 2020. [CrossRef]

7. Jović, M.; Tijan, E.; Žgaljić, D.; Aksentijević, S. Improving Maritime Transport Sustainability Using Blockchain-Based Information Exchange. Sustainability 2020, 12, 8866. [CrossRef]

8. Dutta, P.; Choi, T.-M.; Somani, S.; Butala, R. Blockchain technology in supply chain operations: Applications, challenges and research opportunities. Transp. Res. Part E Logist. Transp. Rev. 2020, 142, 102067. [CrossRef] [PubMed]

9. Van Eck, N.; Waltman, L. Software survey: VOSviewer, a computer program for bibliometric mapping. Scientometrics 2009, 84, 523-538. [CrossRef]

10. Aria, M.; Cuccurullo, C. bibliometrix: An R-tool for comprehensive science mapping analysis. J. Informetr. 2017, 11, 959-975. [CrossRef]

11. Cachin, C.; Sorniotti, M.V.; Weigold, T. Blockchain, Cryptography, and Consensus; IBM Research: Zürich, Switzerland, 2016; Volume 2016.

12. Xiao, Y.; Zhang, N.; Lou, W.; Hou, Y.T. A survey of distributed consensus protocols for blockchain networks. IEEE Commun. Surv. Tutor. 2020, 22, 1432-1465. [CrossRef]

13. Huang, J.; Lei, K.; Du, M.; Zhao, H.; Liu, H.; Liu, J.; Qi, Z. Survey on blockchain incentive mechanism. In International Conference of Pioneering Computer Scientists, Engineers and Educators; Springer: Berlin/Heidelberg, Germany, 2019. 
14. Kang, J.; Xiong, Z.; Niyato, D.; Wang, P.; Ye, D.; Kim, D.I. Incentivizing consensus propagation in proof-of-stake based consortium blockchain networks. IEEE Wirel. Commun. Lett. 2018, 8, 157-160. [CrossRef]

15. Yang, C.-S. Maritime shipping digitalization: Blockchain-based technology applications, future improvements, and intention to use. Transp. Res. Part E Logist. Transp. Rev. 2019, 131, 108-117. [CrossRef]

16. Pedersen, A.B.; Risius, M.; Beck, R. A ten-step decision path to determine when to use blockchain technologies. MIS Q. Exec. 2019, 18, 99-115. [CrossRef]

17. Jabbar, K.; Bjørn, P. Infrastructural grind: Introducing blockchain technology in the shipping domain. In Proceedings of the 2018 ACM Conference on Supporting Groupwork, Sanibel Island, FL, USA, 7-10 January 2018.

18. Tan, A.W.K.; Zhao, Y.; Halliday, T. A blockchain model for less container load operations in China. Int. J. Inf. Syst. Supply Chain Manag. (Ijisscm) 2018, 11, 39-53. [CrossRef]

19. Hasan, H.; Alhadhrami, E.; Aldhaheri, A.; Salah, K.; Jayaraman, R. Smart contract-based approach for efficient shipment management. Comput. Ind. Eng. 2019, 136, 149-159. [CrossRef]

20. Czachorowski, K.; Solesvik, M.; Kondratenko, Y. The Application of blockchain technology in the maritime industry. In Green IT Engineering: Social, Business and Industrial Applications; Springer: Berlin/Heidelberg, Germany, 2019; pp. 561-577.

21. Allen, D.W.; Berg, C.; Davidson, S.; Novak, M.; Potts, J. International policy coordination for blockchain supply chains. Asia Pac. Policy Stud. 2019, 6, 367-380. [CrossRef]

22. Xu, L.; Chen, L.; Gao, Z.; Chang, Y.; Iakovou, E.; Shi, W. Binding the physical and cyber worlds: A blockchain approach for cargo supply chain security enhancement. In Proceedings of the 2018 IEEE International Symposium on Technologies for Homeland Security (HST), Woburn, MA, USA, 23-24 October 2018; IEEE: New York, NY, USA, 2018.

23. Bavassano, G.; Ferrari, C.; Tei, A. Blockchain: How shipping industry is dealing with the ultimate technological leap. Res. Transp. Bus. Manag. 2020, 34, 100428. [CrossRef]

24. Wang, S.; Qu, X. Blockchain applications in shipping, transportation, logistics, and supply chain. In Smart Transportation Systems 2019; Springer: Berlin/Heidelberg, Germany, 2019; pp. 225-231.

25. Jović, M.; Filipović, M.; Tijan, E.; Jardas, M. A Review of Blockchain Technology Implementation in Shipping Industry. Pomorstvo 2019, 33, 140-148. [CrossRef]

26. Todd, P. Electronic bills of lading, blockchains and smart contracts. Int. J. Law Inf. Technol. 2019, 27, 339-371. [CrossRef]

27. Peronja, I.; Lenac, K.; Glavinović, R. Blockchain technology in maritime industry. Pomorstvo 2020, 34, 178-184. [CrossRef]

28. Segers, L.; Ubacht, J.; Tan, Y.-H.; Rukanova, B.D. The use of a blockchain-based smart import declaration to reduce the need for manual cross-validation by customs authorities. In Proceedings of the 20th Annual International Conference on Digital Government Research, Dubai, UAE, 18-20 June 2019.

29. Duru, O.; Imran, M. Blockchain Roaming in the Maritime Industry. 2019. Available online: http://www.okanduru.com/ Blockchain_Roaming_in_the_Maritime_Industry.pdf (accessed on 20 November 2020).

30. Perkušić, M.; Jozipović, Š.; Piplica, D. The Need for Legal Regulation of Blockchain and Smart Contracts in the Shipping Industry. Trans. Marit. Sci. 2020, 9, 365-373. [CrossRef]

31. Tan, W.K.A.; Sundarakani, B. Assessing Blockchain Technology application for freight booking business: A case study from Technology Acceptance Model perspective. J. Glob. Oper. Strateg. Sourc. 2020. [CrossRef]

32. Komathy, K. Verifiable and authentic distributed blockchain shipping framework for smart connected ships. J. Comput. Theor. Nanosci. 2018, 15, 3275-3281. [CrossRef]

33. Rahimi, P.; Khan, N.D.; Chrysostomou, C.; Vassiliou, V.; Nazir, B. A Secure Communication for Maritime IoT Applications Using Blockchain Technology. In Proceedings of the 2020 16th International Conference on Distributed Computing in Sensor Systems (DCOSS), Marina del Rey, CA, USA, 25-27 May 2020; IEEE: New York, NY, USA, 2020.

34. Pitropakis, N.; Logothetis, M.; Andrienko, G.; Stefanatos, J.; Karapistoli, E.; Lalmbrinoudakis, C. Towards the Creation of a Threat Intelligence Framework for Maritime Infrastructures. In Computer Security; Springer: Berlin/Heidelberg, Germany, 2019; pp. 53-68.

35. Petković, M.; Vujović, I. Blockchain security of autonomous maritime transport. J. Appl. Eng. Sci. 2019, 17, 333-337. [CrossRef]

36. Kermani, M.; Parise, G.; Shirdare, E.; Martirano, L. Transactive Energy Solution in a Port's Microgrid based on Blockchain Technology. In Proceedings of the 2020 IEEE International Conference on Environment and Electrical Engineering and 2020 IEEE Industrial and Commercial Power Systems Europe (EEEIC/I\&CPS Europe), Madrid, Spain, 9-12 June 2020; IEEE: New York, NY, USA, 2020.

37. Maritime by Holland. Port of Rotterdam Wants to be the Smartest; Maritime by Holland: Rotterdam, The Netherlands, 2018; pp. 56-58.

38. Hafizon, M.I.; Wicaksono, A.; Farizan, F.N. E-Toll Laut: Blockchain Port as the Key for Realizing Indonesia's Maritime Fulcrum. In Proceedings of the 12th International Conference on Theory and Practice of Electronic Governance, Melbourne, Australia, 3-5 April 2019.

39. Wang, S.; Zhen, L.; Xiao, L.; Alttard, M. Data-Driven Intelligent Port Management Based on Blockchain. Asia-Pac. J. Oper. Res. 2020, 2040017. [CrossRef]

40. Henesey, L.; Lizneva, Y.; Anwar, M. A multi-agent system with blockchain for container stacking and dispatching. In Proceedings of the 21st International Conference on Harbor, Maritime and Multimodal Logistics Modeling and Simulation, HMS, Lisbon, Portugal, 18-20 September 2019; Dime University of Genoa: Genoa, Italy, 2019. 
41. Pranav, P.; Saikiran, A.; Mukul, M.; Ravishankar, B.; Shailaja, V. Critical Analysis of International Shipments within Mainstream Blockchain Framework using Industrial Engineering Techniques. In Proceedings of the 2020 International Conference on Mainstreaming Block Chain Implementation (ICOMBI), Bengaluru, India, 21-22 February 2020; IEEE: New York, NY, USA, 2020.

42. Jugović, A.; Bukša, J.; Dragoslavić, A.; Sopta, D. The possibilities of applying blockchain technology in shipping. Pomorstvo 2019, 33, 274-279. [CrossRef]

43. Ho, T.-C.; Hsu, C.-L. An analysis of key factors influencing integration of blockchain into shipping companies in Taiwan. J. Mar. Sci. Technol. 2020, 28, 229-236.

44. Nasih, S.; Arezki, S.; Gadi, T. Blockchain technology impact on the maritime supply chain. In Proceedings of the 4th International Conference on Smart City Applications, Casablanca, Morocco, 2-4 October 2019.

45. Philipp, R.; Prause, G.; Gerlitz, L. Blockchain and Smart Contracts for Entrepreneurial Collaboration in Maritime Supply Chains. Transp. Telecommun. J. 2019, 20,365-378. [CrossRef]

46. Irannezhad, E. Is blockchain a solution for logistics and freight transportation problems? Transp. Res. Procedia 2020, 48, 290-306. [CrossRef]

47. Shi, H.; Wang, X. Research on the development path of blockchain in shipping industry. In Proceedings of the Asia-Pacific Conference on Intelligent Medical 2018 \& International Conference on Transportation and Traffic Engineering 2018, Beijing, China, 29-31 December 2018.

48. Nguyen, S.; Chen, P.S.-L.; Du, Y. Risk identification and modeling for blockchain-enabled container shipping. Int. J. Phys. Distrib. Logist. Manag. 2020. [CrossRef]

49. Greiman, V. Navigating the Cyber Sea: Dangerous Atolls Ahead. In Proceedings of the ICCWS 2019 14th International Conference on Cyber Warfare and Security: ICCWS 2019, Stellenbosch, South Africa, 28 February-1 March 2019; Academic Conferences and Publishing Limited: Cambridge, MA, USA, 2019.

50. Koens, T.; Poll, E. Assessing interoperability solutions for distributed ledgers. Pervasive Mobile Comput. 2019, 59, 101079. [CrossRef]

51. Buterin, V. Chain Interoperability. R3 Research Paper. 2016. Available online: https://www.r3.com/wp-content/uploads/2017 /06/chain_interoperability_r3.pdf (accessed on 20 November 2020).

52. Brown, R.G. The Five Ingredients of Blockchain Interoperability. 2020. Available online: https://www.r3.com/blog/the-fiveingredients-of-blockchain-interoperability/ (accessed on 23 November 2020).

53. Corda Documentation. What is a CorDapp? 2020. Available online: https://docs.corda.net/docs/corda-os/4.6/cordappoverview.html\#cordapp-components (accessed on 23 November 2020).

54. Accenture \& Fujitsu. Hyperledger Cactus Whitepaper. 2020. Available online: https://github.com/hyperledger/cactus/blob/ master/whitepaper/whitepaper.md (accessed on 23 November 2020).

55. Kaska, M.; Tolga, A.C. Blockchain Software Selection for a Maritime Organization with MCDM Method. In International Conference on Intelligent and Fuzzy Systems; Springer: Berlin/Heidelberg, Germany, 2020.

56. Jagati, S. Blockchain Interoperability: The Holy Grail for Cross-Chain Deployment. 2020. Available online: https:/ / cointelegraph. $\mathrm{com} /$ news/blockchain-interoperability-the-holy-grail-for-cross-chain-deployment (accessed on 23 November 2020).

57. Casino, F.; Dasaklis, T.K.; Patsakis, C. A systematic literature review of blockchain-based applications: Current status, classification and open issues. Telemat. Inform. 2019, 36, 55-81. [CrossRef]

58. Morris, N. ISO Blockchain Standards Planned for 2021. 2018. Available online: https://www.ledgerinsights.com/iso-blockchainstandards / (accessed on 23 November 2020).

59. Gramoli, V.; Staples, M. Blockchain Standard: Can We Reach Consensus? IEEE Commun. Stand. Mag. 2018, 2, 16-21. [CrossRef]

60. European Commission. Joining Forces for Blockchain Standardisation Event Report. 2020. Available online: https://ec.europa. eu/newsroom/dae/document.cfm?doc_id=68644 (accessed on 23 November 2020).

61. Rocket, T.; Yin, M.; Sekniqi, K.; van Renesse, R.; Sirer, E.G. Scalable and probabilistic leaderless bft consensus through metastability. arXiv 2019, arXiv:1906.08936.

62. Sirer, E.G. Open and Programmable Finance with Avalanche. In BlockChainJam 2020; 2020; Available online: https://www. youtube.com/watch?v=Q4XLzTa5G64 (accessed on 23 November 2020).

63. Hirata, E.; Lambrou, M.; Watanabe, D. Blockchain technology in supply chain management: Insights from machine learning algorithms. Marit. Bus. Rev. 2020. [CrossRef]

64. GDPR. General Data Protection Regulation GDPR. 2020. Available online: https://gdpr-info.eu/ (accessed on 23 November 2020).

65. Chainstack. Enterprise Blockchain Protocols Evolution Index 2020. 2020. Available online: https://chainstack.com/wp-content/ uploads / 2020/01/Enterprise-Blockchain-Protocols-Evolution-Index-2020.pdf (accessed on 23 November 2020).

66. Zhou, Y.; Soh, Y.S.; Loh, H.S.; Yuen, K.F. The key challenges and critical success factors of blockchain implementation: Policy implications for Singapore's maritime industry. Mar. Policy 2020, 122, 104265. [CrossRef] [PubMed]

67. Gkerekos, C.; Theotokatos, G.; Bujorianu, L. Digitalisation in the uk maritime sector: A stakeholders'pulse check. In Proceedings of the Marine Industry 4.0, Rotterdam, The Netherlands, 5 November 2019.

68. Gauld, R. Principal-Agent Theory of Organizations. In Global Encyclopedia of Public Administration, Public Policy, and Governance; Springer: Cham, Switzerland, 2016. 
69. Treiblmaier, H. The impact of the blockchain on the supply chain: A theory-based research framework and a call for action. Supply Chain Manag. Int. J. 2018, 23, 15. [CrossRef]

70. Cuypers, I.; Hennart, J.-F.; Silverman, B.S.; Ertug, G. Transaction Cost Theory: Past Progress, Current Challenges, and Suggestions for the Future. Acad. Manag. Ann. 2020, 15, 111-150. [CrossRef]

71. Roeck, D.; Sternberg, H.; Hofmann, E. Distributed ledger technology in supply chains: A transaction cost perspective. Int. J. Prod. Res. 2020, 58, 2124-2141. [CrossRef]

72. Barney, J. Firm resources and sustained competitive advantage. J. Manag. 1991, 17, 99-120. [CrossRef] 